\title{
Effect of unexpected increase in stimulus intensity on reaction time of hand withdrawal'
}

\author{
ARJUN P. PUROHIT \\ QUEEN'S UNIVERSITY, KINGSTON, ONTARIO
}

Latencies of hand withdrawal to a weak stimulus (RTw) and to a strong stimulus, the intensity of which was increased unexpectedly (RTs) were obtained from 32 male and $32 \mathrm{fe}$ male Ss. Most Ss showed facilitatory effect in their RTs. This and other results have been discussed with reference to the findings of an earlier study in. which $S$ s were required to press a reaction time key in the same situation. A modification of curvilinear theory of performance has been suggested.

In a recent paper (Purohit, 1966) it was reported that Ss whose reaction time to a weak stimulus (RTw) was long, showed facilitatory effect (RTs - RTw =-) in their reaction time to a strong stimulus (RTs), the intensity of which was increased unexpectedly. Conversely, Ss whose RTw was short showed inhibitory effect (RTs $-\mathrm{RTw}=+$ ) in their RTs in the same situation. We gave a number of reasons why an interpretation of these results in terms of curvilinear performance theory of activation was not tenable. Instead, an alternate explanation was proposed which can be summarized as follows: unexpected increase in stimulus intensity produces stronger facilitatory and weaker inhibitory effects on the RTs of persons whose RTw is long; it produces inhibitory and facilitatory effects of equal strength in the RTs of persons whose $\mathrm{RTw}$ is of medium duration; and it produces weaker facilitatory and stronger inhibitory effects on the RTs of persons whose RTw is short.

In the present study, it has been attempted to locate the source of inhibitory effects on the RTs in this situation. In the previous study, the Ss were required to press a reaction time key as soon as they heard the auditory stimulus. It is well known that startle produced by an unexpectedly given strong auditory stimulus causes flexion of limbs (Landis \& Hunt, 1939). We suspect that this tendency to withdraw might have been the source of inhibitory effect. In this experiment the Ss were required to withdraw at the onset of the stimulus. Therefore, the withdrawal tendency resulting from the unexpected intensity of the stimulus would facilitate, instead of inhibit, the speed of reaction time. Following this line of argument, we had three hypotheses: (1) no inhibitory effect will be noticed in the Ss' RTs irrespective of the duration of their RTw; (2) with the source of inhibitory effect converted into that of facilitatory effect, the amount of facilitatory effect shown in this experiment will be much larger than that in the previous experiment; and (3) the regression line of RTs-RTw to the RTw in this experiment will be parallel to the one obtained in the previous experiment.

\section{Method}

Thirty-two male and 32 female students of Queen's University volunteered to act as Ss in this experiment.

The Ss were asked to place the index finger of their preferred hand on a 1/2-in. diameter brass disk and withdraw their finger from it as soon as they heard a sound through their earphone. All the auditory stimuli were $500 \mathrm{cps}$ and of $1 / 2-\mathrm{sec}$. duration. The first three stimuli were of $75 \mathrm{~dB}$ and the fourth of $120 \mathrm{~dB}$. The Ss were not advised of the change in the intensity. The stimuli were given in 30 to $40 \mathrm{sec}$. intervals. The reaction time was recorded by a timer which was started by the auditory stimuli and stopped by the loss of contact from the brass disk. The Ss were asked not to discuss the experiment with others.

Results

As in the previous experiment, three response measures were taken into consideration: reaction time to the third weak stimulus (RTw), reaction time to the strong stimulus (RTs) and inhibition-facilitation score (RTs $R T w)$. No sex difference was noticed in either RTw $(t=0.32, d f=62)$ or RTs $(t=0.39, d f=62)$. All the 64 Ss were serially ordered in terms of the duration of RTw and divided into eight equal groups. Figure 1 shows the relationship between RTw and -RTs -RTw found in this present and the previous experiment indicated by inward response and forward response regression lines respectively. Our first hypothesis was confirmed since the RTs-RTw scores of all the eight groups were facilitatory. On closer examination, 58 or $94 \%$ of the $\mathrm{Ss}$, instead of predicted $100 \%$, were facilitators. However,

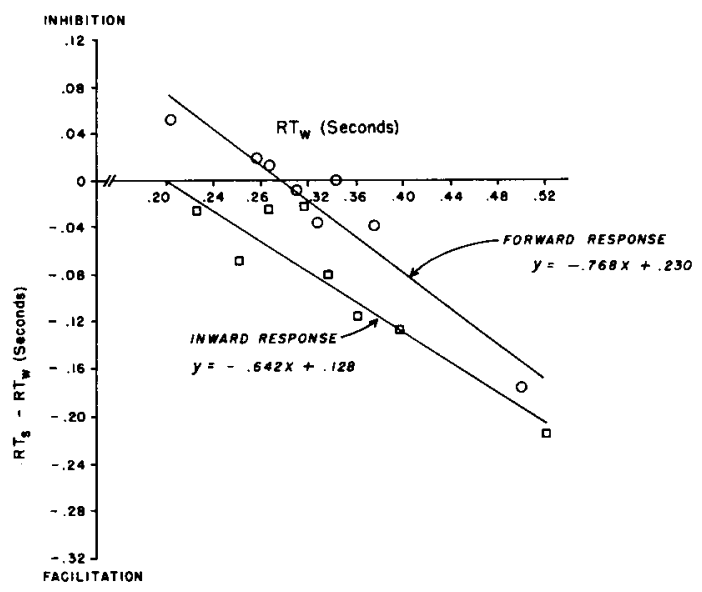

Fig. 1. Relationship between $\mathbf{R T}_{\mathbf{W}}$ and RTs-RTW obtained for forward response (Purohit, 1966) and inward response in this experiment. See text for details. 
the difference between the predicted and obtained percentages of facilitators was not significant $(t=.68$, $\mathrm{df}=62$ ). The second hypothesis was also confirmed because, as can be seen in Fig. 1, the inward regression line was always towards the facilitatory side of the forward response regression line obtained from the previous experiment. This difference is statistically significant $(t=5.43, d f=190, p<.001)$. Our third hypothesis predicted that both the regression lines would be parallel. Figure 1 shows that the slopes of the two lines are quite similar, if not completely identical.

We also found that the correlation between RTw and RTs - RTw was - .72 compared with - .32 in the previous study. Furthermore, the correlation between RTw and RTs was +.44 compared with +.225 in the previous study. Discussion

Since the hypotheses were sufficiently confirmed, the suspicion regarding the withdrawing tendency caused by the startle response as the source of this inhibitory effect on forward response in the previous experiment seems to be well founded. This study also indicates more clearly why interpretation of results of the previous study in terms of the curvilinear performance theory of activation is inadequate. Sudden rise in activation does not necessarily impede performance per se. On the other hand, it appears that facilitatory and inhibitory effects of high activation on performance are very much conditional to the nature of the task. If the task demands behavior which is in harmony with the activation produced tendency then high activation is facilitatory. On the other hand, if it demands behavior opposite to the activation produced tendency then high activation may have an inhibitory effect on performance.

\section{References}

Landis, C., \& Hunt, W. A. The startle pattern. New York: Farber and Rinehart, Inc., 1939.

Purohit, A. P. Some correlates of inhibitory-facilitatory effect on reaction-time due to unexpected increase in stimulus intensity. Psychon. Sci., 1966, 5, 53-54.

\section{Note}

1. This research was financed by National Research Council of Canada. 